\title{
Timing of Endotracheal Intubation and Mortality among Patients with Severe COVID-19
}

\author{
Banambar Ray ${ }^{1} \odot$, Arun K Sahu² ${ }^{\infty}$ \\ Indian Journal of Critical Care Medicine (2021): 10.5005/jp-journals-10071-23834
}

\section{BACKGROUND}

Coronavirus disease-2019 (COVID-19) pneumonia causing severe acute respiratory distress syndrome (ARDS) is different from other causes of severe ARDS in that it is associated with near normal lung compliance. Severe hypoxia of COVID-19 presents differently in different patients. ${ }^{1}$ Patients may be normally breathing (happy hypoxia or silent hypoxia), or they could be in severe acute respiratory syndrome (called SARS-COVID-19) and therefore dyspneic. The L and $\mathrm{H}$ phenotypes (low elastance and high elastance, respectively) described by Gattinoni et al. are the basis of instituting various modes of oxygenation and ventilation. Use of high-flow nasal cannula (HFNC), continuous positive airway pressure, and noninvasive ventilation (NIV) in L-type dyspneic patients, even if they have deteriorated to $\mathrm{H}$ type, has delayed invasive mechanical ventilation in SARS-COVID-19 patients. In an editorial, Navas-Branco and Dudaryk suggested that efforts should be focused to avoid intubation and mechanical ventilation in severe COVID-19 patients. ${ }^{2}$ However, more and more discovery of patient self-inflicted lung injury (P-SILI) during spontaneous breathing in COVID-19 patients makes a case for early invasive mechanical ventilation. ${ }^{3}$ Timing of endotracheal intubation (simply called intubation), early or late, in severe respiratory distress of COVID-19 patients continues to be debated in so far as in-hospital mortality is concerned. Few retrospective single or multicentered studies have been published with different results.

\section{Current Evidence}

In a retrospective cohort study, out of 231 severe COVID-19 patients admitted to the intensive care unit (ICU), 109 (47.2\%) were given HFNC and 97 (42\%) were intubated straight away. ${ }^{4}$ Out of 109 on HFNC, 78 (71.6\%) were eventually intubated making the total of 175 who were intubated and ventilated. Timing of intubation was as follows: 76 (43.4\%) within 8 hours of admission, 57 (32.6\%) between 8 hours and 24 hours, and 42 (24\%) equal to or beyond 24 hours. There was no difference in mortality, duration of mechanical ventilation, and ICU length of stay among the three groups. A multicentered retrospective observational study was done in 47 patients of severe COVID-19. ${ }^{5}$ Twenty-three (46.9\%) patients were intubated on the day they fulfilled ARDS criteria (early), and 24 (51.1\%) were initially not intubated ( 8 were not intubated at all). While 21 patients died, there was no statistical difference in deaths between the two groups. In a study from Evangelismos Hospital, Athens, Greece, Stempos et al. reported 101 consecutively hospital admitted cases of COVID-19. ${ }^{6}$ Patients were stratified into two groups (early intubation
1,2Department of Critical Care Medicine, Sum Ultimate Medicare, Bhubaneswar, Odisha, India

Corresponding Author: Department of Critical Care Medicine, Sum Ultimate Medicare, Bhubaneswar, Odisha, India, Phone: +91 9238310648, e-mail: banambar.ray@gmail.com

How to cite this article: Ray B, Sahu AK. Timing of Endotracheal Intubation and Mortality among Patients with Severe COVID-19. Indian J Crit Care Med 2021;25(5):477-478.

Source of support: Nil

Conflict of interest: None

and delayed or no intubation groups). The delayed or no intubation group comprised of patients who were on non-rebreathing oxygen or HFNC for more than or equal to 24 hours or on NIV for any length of time for avoiding intubation. Fifty-nine (58\%) patients were in general wards, and forty-two (41.58\%) patients were admitted in ICU. Intubation was decided based on the hemodynamic instability, altered mentation, and respiratory distress and not on hypoxia without respiratory distress. Thirty-six (86\%) patients were intubated, and ICU mortality was eleven (26\%) overall. Fourteen patients had early intubation (within 48 hours of ICU admission) with mortality of three (21\%), and eighteen patients had late intubation (48 hours or more) with mortality of eight (33\%), suggesting that "Early intubation (as opposed to delayed or no intubation) was not associated with mortality even after adjustment for sex." This can be construed as early intubation was associated with lesser mortality even though it was not statistically significant. An observational study from Philadelphia compared early versus late intubation in COVID-19 patients (at admission or within 2 days of a documented $\mathrm{FiO}_{2} \geq 0.5$ was early intubation). ${ }^{7}$ Early intubation group had a statistically significant higher age and SOFA score. They concluded that the timing of intubation had not influenced the outcome. Even they suggested that "a trial of noninvasive strategies of oxygenation in an attempt to avoiding intubation might not be harmful."

In an earlier retrospective cohort study (January 30, 2020 to April $30,2020)$ in five hospitals in New York City, it was concluded that early intubation may be associated with improved survival. ${ }^{8}$ The authors even went a step deeper to say that the adjusted hazard ratio for mortality was $1.03(95 \% \mathrm{Cl}, 1.01-1.05)$ for each day of delay in intubation. They were, however, unable to determine whether only delayed intubation resulted in the observed increase in mortality. They also revealed that duration of mechanical ventilation greater than 3-4 weeks was associated with a lower risk of in-hospital death relative to those extubated earlier. They advised that delay

(c) Jaypee Brothers Medical Publishers. 2021 Open Access This article is distributed under the terms of the Creative Commons Attribution 4.0 International License (https://creativecommons.org/licenses/by-nc/4.0/), which permits unrestricted use, distribution, and non-commercial reproduction in any medium, provided you give appropriate credit to the original author(s) and the source, provide a link to the Creative Commons license, and indicate if changes were made. The Creative Commons Public Domain Dedication waiver (http://creativecommons.org/publicdomain/zero/1.0/) applies to the data made available in this article, unless otherwise stated. 
in intubation in COVID-19-related acute respiratory failure should be undertaken with caution.

While one is seized with early intubation or late intubation, is there a possibility that no intubation could be better than intubation in COVID-19 patients? The retrospective cohort study in six different ICUs in Emory University School of Medicine, Atlanta, Georgia, revealed that among those who were given HFNC, even though $71.6 \%$ eventually received invasive ventilation, $28.4 \%$ still could avoid intubation without increasing mortality. ${ }^{4}$ It suggests that one need not hurry for intubation in such patients while trying alternative modes of oxygenation if clinical judgment permits.

\section{The Study in the Current Issue}

The Indian study published in this issue is a retrospective observational study taken up between April and October 2020 included 147 intubated COVID-19 patients. They are stratified into early and late (within and beyond 48 hours of ICU admission, respectively). It is observed that patients in the early intubation group had statistically significant lesser mortality (60\% vs $77.7 \%)$ despite having higher APACHE II and lower absolute lymphocyte count. The authors have rightly suggested to go for a larger randomized prospective study to validate the findings of this retrospective analysis.

\section{Author's View}

Intubation is a clinical decision and is generally individualized. COVID-19 pneumonia and ARDS are different in many aspects from ARDS of other etiologies. Decision to intubate is further influenced in a pandemic situation because of logistic, resource, and social issues, particularly in India. All the studies so far we come across are retrospective in nature and therefore have many deficiencies. In COVID-19 pandemic, taking up a prospective study on the basis of the timeline for intubation does not seem appropriate because that might cause a bias and even may be ethically and clinically not correct. So, it can be suggested that a system of written-down protocol-based approach and documentation should be followed, and later data compiled and analyzed by independent observers. ${ }^{9,10}$

\section{ORCID}

Banambar Ray @ https://orcid.org/0000-0002-8711-1867

Arun K Sahu $\odot$ https://orcid.org/0000-0002-1459-2508

\section{References}

1. Gattinoni L, Chiumello D, Caironi P, Busana M, Romitti F, Brazzi L, et al. Covid 19 pneumonia: different respiratory treatments for different phenotypes? Intensive Care Med 2020;46(6):1099-1102. DOI: 10.1007/ s00134-020-06033-2.

2. Navas-Blanco JR, Dudaryk R. Management of respiratory distress syndrome due to Covid-19 infection. BMC Anaesthesiol 2020;20:177. DOI: 10.1186/s12871-020-01095-7.

3. Grieco DL, Menga LS, Eleuteri D, Antonelli M. Patient selfinflicted lung injury: implications for acute hypoxaemic respiratory failure and ARDS patients on non-invasive support. Minerva Anaestesiol 2019;85:1014-1023. DOI: 10.23736/s03759393.19.13418-9.

4. Alfonso $\mathrm{CH}$, Max WA, Maxwel AH, Robichaux CJ, Edwards JA, Fazio JC, et al. Timing of intubation and mortality among critically ill coronavirus disease 2019 patients: a single-center cohort study. Crit Care Med 2020;48:e1045-e1053. DOI: 10.1097/ CCM.0000000000004600.

5. Lee YH, Choi KJ, Choi SH, Lee SY, Kim KC, Kim EJ, et al. Clinical significance of timing of intubation in critically ill patients of Covid-19: a multi-center retrospective study. J Clin Med 2020;9:2847. DOI: 10:3390/jcm9092847.

6. Stempos II, Xourgia E, Ntaidou TK, Zervakis D, Magira EE, Kotanidou A, et al. Effect of early vs delayed or no intubation on clinical outcomes of patients with Covid-19: an observational study. Front Med 2020;7:614152. DOI: 10.3389/fmed.2020.614152.

7. Matta A, Chaudhary S, Bryan L, DeJoy R, Gul F, Torres R, et al. Timing of intubation and its implications on outcomes in critically ill patients with coronavirus disease 2019 infection. Crit Care Explorat 2020;2(10):e0262. DOI: 10.1097/ CCE.0000000000000262.

8. Hyman JB, Leibner ES, Tandon P, Egorova NN, BassilyMarcus A, Kohli-Seth R, et al. Timing of intubation and in-hospital mortality in patients with coronavirus disease 2019. Crit Care Explorat 2020;2(10):e0254. DOI: 10.1097/ CCE.0000000000000254

9. Zirpe KG, Tiwari AM, Gurav SK, Deshmukh AM, Suryawanshi PB, Wankhede PP, et al. Timing of Invasive Mechanical Ventilation and Mortality among Patients with Severe COVID-19-associated Acute Respiratory Distress Syndrome (CARDS). Indian J Crit Care Med 2021;25(5):493-498.

10. Zirpe KG, Tiwari AM, Gurav SK, Deshmukh AM, Suryawanshi PB, Wankhede PP, et al. Timing of Invasive Mechanical Ventilation and Mortality among Patients with Severe COVID-19-associated Acute Respiratory Distress Syndrome. Indian J Crit Care Med 2021;25(5):493-498 